\title{
MicroRNA-199a-3p is downregulated in gastric carcinomas and modulates cell proliferation
}

\author{
W. Peng ${ }^{1 *}$, Z.-Y. Chen ${ }^{1 *}$, L. Wang ${ }^{2}$, Z. Wang ${ }^{3}$ and J. Li ${ }^{1}$ \\ ${ }^{1}$ Department of Medical Oncology, Fudan University Shanghai Cancer Center; \\ Department of Oncology, Shanghai Medical College, Fudan University, \\ Shanghai, China \\ ${ }^{2}$ Department of Oncology, Affiliated First People's Hospital, \\ Shanghai Jiao Tong University, Shanghai, China \\ ${ }^{3}$ Shanghai ChemPartner Inc., Shanghai, China \\ *These authors contributed equally to this study. \\ Corresponding author: J. Li \\ E-mail: fudanlijin@163.com / lglou@mail.shcnc.ac.cn
}

Genet. Mol. Res. 12 (3): 3038-3047 (2013)

Received January 15, 2013

Accepted June 14, 2013

Published August 20, 2013

DOI http://dx.doi.org/10.4238/2013.August.20.5

\begin{abstract}
MicroRNAs (miRNAs) are small non-coding RNAs that regulate the translation of targeted mRNAs. An increasing amount of evidence indicates that miRNAs play important role in cancer pathogenesis, apoptosis, proliferation, and differentiation as oncogenes or tumor suppressors. Recently, miRNA-199a has been shown to be involved in many human cancers, although the role of miRNA-199a$3 p$ in gastric cancer has not yet been evaluated. In the present study, the expression of miRNA-199a-3p was found to be significantly downregulated in human gastric cancer tissues and cells. miRNA-199a$3 p$ induced anti-proliferation effects on human gastric cancer cells. Furthermore, using quantitative RT-PCR (real-time polymerase chain reaction) and luciferase reporter assays, $m T O R$ was identified as a direct target gene of miRNA-199a-3p that is downregulated by it. In conclusion, our findings suggest that miRNA-199a-3p is associated with human gastric cancer through its ability to decrease cancer cell proliferation and target the mTOR signaling pathway, and, therefore, may provide a novel
\end{abstract}


therapeutic target for the treatment of human gastric cancer.

Key words: miRNA-199a-3p; Gastric cancer; mTOR; Anti-proliferation

\section{INTRODUCTION}

Gastric cancer, one of the most common malignant diseases, remains the second most frequent cause of cancer-related deaths in the world (Ichimi et al., 2009; Tsukamoto et al., 2010; Ueda et al., 2010; Tsujiura et al., 2010). Approximately 7,000,000 people die from gastric cancer annually. Although improvements in diagnostic techniques and peri-operative treatments have resulted in better clinical outcomes and a decreased mortality rate for patients with early gastric cancer, the prognosis is still very poor for patients with advanced disease at diagnosis (Martin et al., 2002; Hohenberger and Gretschel, 2003). An understanding of the molecular mechanisms of gastric cancer and new therapeutic countermeasures, therefore, are critically needed to improve the survival rates of patients with gastric cancer.

Recent studies have found that microRNAs (miRNAs) act as potent oncogenes and tumor suppressor genes in human cancers (Kent and Mendell, 2006; Dyrskjøt et al., 2009; Gandellini et al., 2009; Su et al., 2009). miRNAs are small non-coding, single-stranded RNA molecules of 18-25 nucleotides in length that are cleaved from 70-100 nucleotide hairpin pre-miRNA precursors (Bartel, 2004). Mature miRNAs regulate gene expression by binding to the 3'-untranslated region (UTR) of targeted mRNAs, which represses their translation (Bartel, 2004). Bioinformatic analysis has revealed that nearly one-third of all human genomic mRNAs are targeted by miRNA (Lewis et al., 2005; Garzon et al., 2009). Furthermore, studies have found that miRNAs control many cellular processes including development, proliferation, the stress response, apoptosis, cell cycle progression, and differentiation (Ambros, 2004; Lim et al., 2005; Engels and Hutvagner, 2006; Bartel, 2004, 2009). Other evidence demonstrated that miRNAs are aberrantly expressed or mutated in many cancers, suggesting that the primary functions of miRNAs are as oncogenes or tumor suppressors (Bentwich et al., 2005; Zamore and Haley, 2005). For example, the expression of miRNA let-7 is decreased in human lung carcinomas (Iorio et al., 2005). Moreover, the expression of miRNA-34 is reduced in non-small cell lung cancer (NSCLC) and, conversely, the restoration of miRNA-34 expression inhibits the growth of NSCLC (Hatfield and Ruohola-Baker, 2008). The expression of miRNA-143 and -145, on the other hand, is downregulated in colon cancers, and transfection of human gastric MKN-1 cells with miRNA-145 results in growth-inhibitory effects (Takagi et al., 2009). Lastly, miRNA-150 is over-expressed in gastric cancer cell lines and, consequently, miRNA-150 inhibitors suppress the growth of the HeLa and A549 cell lines (Wu et al., 2010). Alterations in miRNA expression, therefore, are highly associated with the development and progression of various human cancers.

Recent investigations have revealed that the expression of miRNA-199a is altered in several human cancers (Yanaihara et al., 2006; Lee et al., 2008; Garzon et al., 2009). Specifically, the expression of miRNA-199a is increased in ovarian cancer cells, pancreatic adenocarcinomas, and cervical carcinomas (Yanaihara et al., 2006; Garzon et al., 2009). Furthermore, miRNA-199a can reduce cellular resistance to cytotoxic drugs (Garzon et al., 2009). The expression of miRNA-199a$3 \mathrm{p}$ is, however, downregulated in liver, breast, and bladder cancer, and promotes the proliferation and survival of endothelial and breast cancer cells (Brennecke et al., 2003; O'Donnell et al., 2005; Jiang et al., 2008). Moreover, the miRNA-199a-3p level is significantly decreased in human os- 
teosarcoma cell lines and, thus, transfection of miRNA-199a-3p mimic into those cells inhibits cell growth and migration (Hatfield and Ruohola-Baker, 2008). Ueda et al. (2010) suggested that miRNA-199a is one of the most important miRNAs involved in the progression of gastric cancer. Owing to these previously described findings, we propose that miRNA-199a-3p functions as a tumor suppressor gene and plays an important role in the clinical pathology of gastric carcinogenesis. In the present study, therefore, we investigated the expression and mechanisms of miRNA-199a-3p in gastric cancer. We found that miRNA-199a-3p expression was downregulated in both human gastric cancer tissues and cells, compared with normal counterparts, and miRNA-199a-3p inhibited the proliferation of human gastric cancer cells. Further, using bioinformatic analysis, we identified $m T O R$ as a direct target gene of miRNA-199a-3p and confirmed this interaction with a reporter construct bearing the 3'-UTR of $m$ TOR mRNA. Our findings, therefore, suggest that miRNA-199a$3 p$ plays a critical role in gastric cancer by targeting the mTOR signaling pathway and provides a candidate for diagnosis and treatment of human gastric cancer.

\section{MATERIAL AND METHODS}

\section{Tissue samples}

Gastric tumor and adjacent normal, non-cancerous tissue samples were surgically obtained from 12 patients at the Department of Oncology of the Cancer Hospital, Fudan University. Tissue samples were gathered in the same manner, snap-frozen immediately in liquid nitrogen, and stored in a deep freezer at $-80^{\circ} \mathrm{C}$. The use of these tissue samples for the experiments outlined below was approved by all patients and by the local Ethics Committee of the Shanghai Cancer Center of the Fudan University.

\section{Cell culture and transfection}

The human gastric epithelial cell line GES-1 and the human gastric cancer cell lines AGS and SGC-7901 were purchased from the American Type Culture Collection. Cells were grown at $37^{\circ} \mathrm{C}$ with $5 \% \mathrm{CO}_{2}$ saturated humidity, and cultured in Dulbecco's modified Eagle's medium (Gibco, USA) supplemented with $100 \mathrm{U} / \mathrm{mL}$ penicillin and $100 \mu \mathrm{g} / \mathrm{mL}$ streptomycin, $2 \mathrm{mM}$ glutamine, and 10\% fetal bovine serum. The miRNA-199a-3p mimic (5'-ACAGUAGUCUGCACAUUGGUUA-3', Ambion, TX, USA), inhibitor (Ambion), and negative control (Ambion) were designed and synthesized by Invitrogen (USA). Cells were transfected with the miRNA-199a-3p mimic, inhibitor, or individual negative control using Lipofectamine 2000 (Invitrogen) according to manufacturer instructions.

\section{Cell proliferation assay}

Cell proliferation was determined with the 3-(4,5-dimethylthiazol-2-yl)-5-(3carboxymethoxyphenyl)-2-(4-sulfophenyl)-2H-tetrazolium inner salt (MTS) assay. GES-1 and AGS cells transfected with the miRNA-199a-3p mimic or inhibitor were seeded with culture medium on 96-well culture plates to an optimal density corresponding to 6 or $2 \times 10^{3}$ cells per well. After $0,24,48$, and $72 \mathrm{~h}$ of incubation, cells were stained with $20 \mu \mathrm{L}$ MTS (317 $\mu \mathrm{g} /$ $\mathrm{mL}$ ) for $4 \mathrm{~h}$ at $37^{\circ} \mathrm{C}$. Absorbance at $490 \mathrm{~nm}$ was then read with a microplate reader (BioRad, 
USA). Each experiment was performed in triplicate and repeated for three replicates.

\section{Quantitative real-time PCR (qRT-PCR)}

Total RNA was extracted from tissue samples and cells cultured using Trizol reagent (Invitrogen) according to manufacturer instructions. Reverse transcription was performed with a TaqMan Reverse Transcription Kit (Applied Biosystems, USA). qRT-PCR assays were carried out using SYBR Green Real-Time PCR Master Mix (Toyobo, Osaka, Japan) and RTPCR amplification equipment (ABI 7500fast), with the specific primers listed in Table 1 . The expression of genes of interest was determined following the normalization of their cycle threshold values to those of the control gene, $\beta$-actin. Mature miRNA-199a-3p expression was measured using a mirVana qRT-PCR miRNA Detection Kit (Ambion), with U6 as an internal control. The optical density of the resultant bands was quantified by the Image-Pro Plus 5.1 software (Media Cybernetics, MD, USA).

\begin{tabular}{|c|c|c|}
\hline Target gene & Primer name & Sequence $\left(5^{\prime}-3^{\prime}\right)$ \\
\hline \multirow[t]{2}{*}{$\beta$-actin } & Sense strand & $\begin{array}{l}\text { CAATGAAGATCAAGATCATTGCTCCTCC } \\
\end{array}$ \\
\hline & Antisense strand & TCAAGAAAGGGTGTAACGCAACTAAGTC \\
\hline \multirow[t]{2}{*}{ mTOR } & Sense strand & ATGCCACGCTCTGGCTACGTG \\
\hline & Antisense strand & CTGGGAGCCCACCTTGAAGGAGT \\
\hline \multirow[t]{2}{*}{ akt } & Sense strand & ATGCCACGCTCTGGCTACGTG \\
\hline & Antisense strand & CTGGGAGCCCACCTTGAAGGAGT \\
\hline \multirow[t]{2}{*}{$p 70$} & Sense strand & ATGCCACGCTCTGGCTACGTG \\
\hline & Antisense strand & CTGGGAGCCCACCTTGAAGGAGT \\
\hline \multirow[t]{2}{*}{ bcl-2 } & Sense strand & ATGCCACGCTCTGGCTACGTG \\
\hline & Antisense strand & CTGGGAGCCCACCTTGAAGGAGT \\
\hline \multirow[t]{2}{*}{ caspase 3} & Sense strand & ATGCCACGCTCTGGCTACGTG \\
\hline & Antisense strand & CTGGGAGCCCACCTTGAAGGAGT \\
\hline
\end{tabular}

\section{mTOR 3'-UTR luciferase reporter assay}

Double-stranded oligonucleotides corresponding to the miRNA-199a-3p binding site in mTOR 3'-UTR were chemically synthesized (Sigma, USA). Their sequences were as follows: forward: 5'-CAATGAAGATCAAGATCATTGCTCCTCC-3', reverse: 5'-TCAAGAAAGGGTGTAACGCAACTAAGTC-3'. Using Lipofectamine 2000 (Invitrogen) according to the protocol provided, AGS cells were transfected on 6-well plates with $2 \mu \mathrm{g}$ mTOR 3'-UTR luciferase reporter psiCHECK plasmid or the control plasmid (Promega, Madison, WI, USA) per well. Cells in each well were also co-transfected with $50 \mathrm{nM}$ miRNA199a-3p mimic, inhibitor, or negative control. Luciferase assays were performed with the Bright-Glo Luciferase Assay System (Promega) $48 \mathrm{~h}$ after transfection.

\section{Statistical analysis}

All statistical analyses were performed using SPSS 10.0. Data are reported as means \pm standard deviation. The statistical correlation of data between groups was determined by oneway analysis of variance and the Student $t$-test, where results with a $\mathrm{P}<0.05$ were considered to be significant. 


\section{RESULTS}

\section{Differing miRNA-199a-3p expression levels between normal and gastric cancer tissues}

To determine the expression of miRNA-199a-3p in gastric cancer tissues, qRT-PCR was used to compare the expression of miRNA-199a-3p between 12 cases of normal and gastric cancer tissue samples. Each tumor and normal sample was derived from a single patient specimen. The expression of miRNA-199a-3p was clearly decreased in gastric cancer tissues when compared with the corresponding non-cancerous tissues for the majority of cases (Figure 1A). Only one patient displayed increased miRNA-199a-3p expression levels in cancer tissues, while in another the expression of miRNA-199a-3p did not change between tissue types (Figure 1A). In most cases, therefore, miRNA-199a-3p in the cancerous tissue had a lower level of expression than in normal tissue.

A

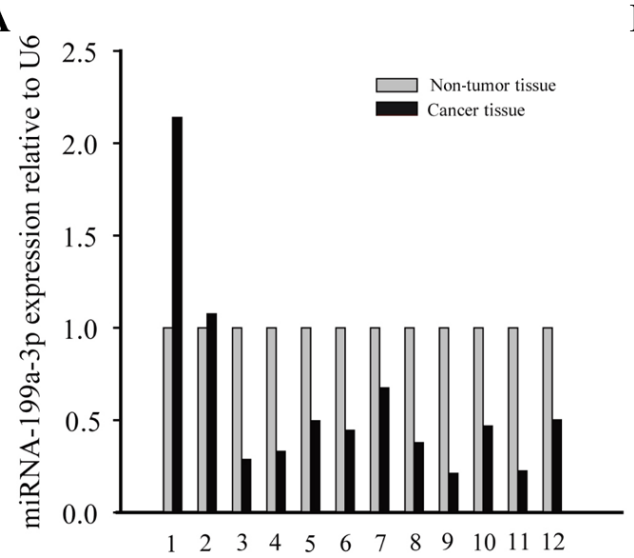

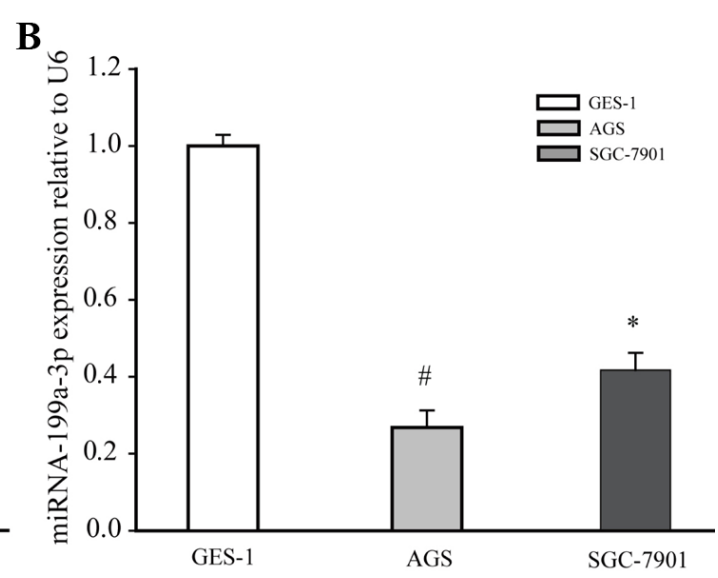

Figure 1. miRNA-199a-3p was downregulated in human gastric cancer tissues and cells. A. Expression of miRNA$199 \mathrm{a}-3 \mathrm{p}$ decreased in human gastric cancer tissue samples compared with the adjacent normal gastric tissues measured by qRT-PCR. B. Expression of miR-139a-3p decreased in human gastric cancer cell lines AGS and SGC7901 compared with the normal gastric epithelium cell line GES-1 measured by qRT-PCR. U6 was used as an internal standard in each group. Data are reported as means $\pm \mathrm{SD}$. ${ }^{*} \mathrm{P}<0.05,{ }^{*} \mathrm{P}<0.001$, compared with GES- 1 cells.

\section{Differing miRNA-199a-3p expression levels in gastric cancer and gastric epithelial cell lines}

To verify the observed expression pattern of miRNA-199a-3p in gastric cancer tissues, miRNA-199a-3p expression was also examined in a gastric cancer cell line and normal gastric epithelial cells. Our findings revealed that the expression level of miRNA-199a-3p was significantly reduced in AGS and SGC-7901 cell lines compared to GES-1 cells (Figure 1B). Specifically, miRNA-199a-3p expression levels were 0.26- $(\mathrm{P}<0.001)$ and 0.43 -fold $(\mathrm{P}<0.01)$ lower in AGS and SGC-7901 cell lines than in the GES-1 cell line, respectively (Figure 1B).

\section{Anti-proliferation effect of miRNA-199a-3p on a gastric cancer cell line}

The observed lower level of miRNA-199a-3p expression in gastric cancer tissues and cell lines indicates that miRNA-199a-3p over-expression would be detrimental to cancer development, 
while miRNA-199a-3p would be favorable. To examine the role of miRNA-199a-3p in cell proliferation, MTS assays were performed to determine cell proliferation activity. We transfected the AGS cell line with miRNA-199a-3p mimic or negative control, or blocked miRNA-199a-3p expression by transient transfection with an inhibitor. Transfection efficiency was confirmed by qRT-PCR. The results revealed that the miRNA-199a-3p expression level was 3-fold higher or 0.5 -fold lower in cells transfected with the miRNA-199a-3p mimic or the inhibitor, respectively, than in cells transfected with the negative control (data not shown). Cell proliferation was evaluated at $0,24,48$, and $72 \mathrm{~h}$ after transfection. The growth of cells transfected with the miRNA-199a$3 p$ mimic was significantly inhibited compared with cells transfected with the negative control or untransfected AGS cells (Figure 2). In contrast, the proliferation of cells transfected with the miRNA-199a-3p inhibitor was obviously induced (Figure 2, P $<0.05$ ). Taken together, therefore, these data suggest that miRNA-199a-3p has an anti-proliferation effect on the gastric cell line.

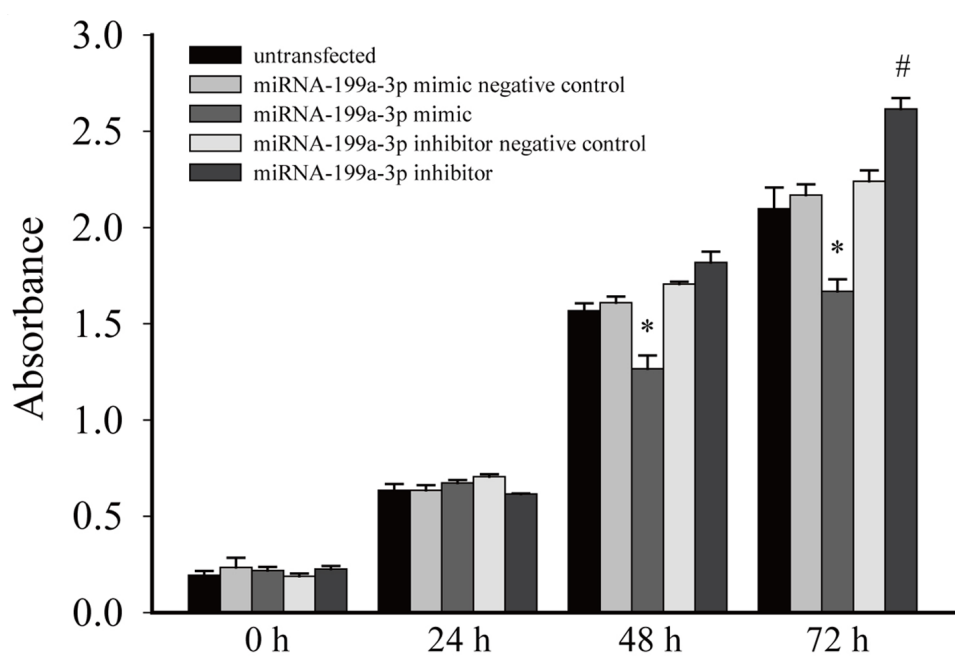

Figure 2. Anti-proliferation effect of miRNA-199a-3p on the growth of human gastric cancer cells. Proliferation of gastric cancer cell was assessed by MTS assays. The growth of gastric cancer cells was detected after 0, 24, 48, and $72 \mathrm{~h}$ of incubation. Data are reported as means $\pm \mathrm{SD} .{ }^{*} \mathrm{P}<0.05$, compared with untransfected cells or cells transfected with the miRNA-199a-3p mimic negative control. ${ }^{\#} \mathrm{P}<0.05$, compared with untransfected cells or cells transfected with the miRNA-199a-3p inhibitor negative control.

\section{Identification of the miRNA-199a-3p target}

To evaluate the molecular mechanism behind the observed anti-proliferation effect of miRNA-199a-3p on a gastric cell line, we subsequently determined the expression of several genes likely involved in cell growth or programmed cell death in the AGS cell line, including akt, bcl-2, caspase 3, p70, and $m T O R$, by qRT-PCR. We found that the mRNA level of mTOR was significantly reduced in AGS cells transfected with the miRNA-199a-3p mimic and enhanced when cells were transfected with the miRNA-199a-3p inhibitor, compared with untransfected cells or cells transfected with the negative control (Figure 3E). The mRNA levels of akt, bcl-2, caspase 3, and p70 did not differ between AGS cells transfected with the miRNA-199a-3p mimic or inhibitor (Figure 3A-D). 

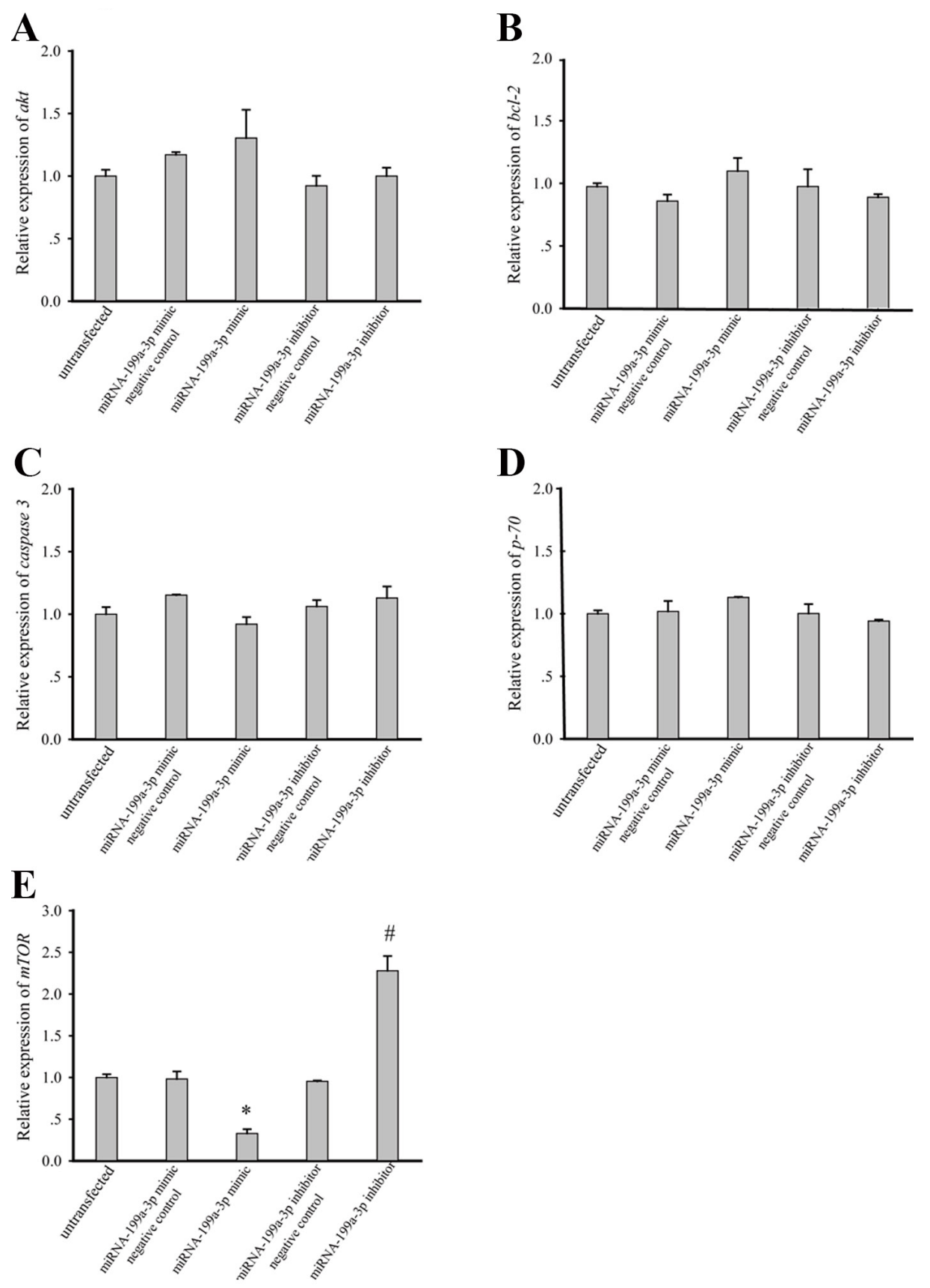

Figure 3. Effect of miRNA-199a-3p on gene expression. The mRNA levels of akt (A), bcl-2 (B), caspase 3 (C), $p-70$ (D), and $m$ TOR (E) were evaluated by qRT-PCR in AGS cells transfected with miRNA-199a-3p mimic or inhibitor. Data are reported as means $\pm \mathrm{SD} .{ }^{*} \mathrm{P}<0.001$, compared with untransfected cells or cells transfected with the miRNA-199a-3p mimic negative control. ${ }^{\#} \mathrm{P}<0.001$, compared with untransfected cells or cells transfected with the miRNA-199a-3p inhibitor negative control. 


\section{$m T O R$ is a direct target of miRNA199a-3p}

To determine whether or not $m$ TOR was directly targeted by miRNA-199a-3p, the TargetScan was first used to identify $m T O R$ as a putative target of miRNA-199a-3p. A luciferase reporter gene was then linked to the $m T O R$ 3'-UTR within the psiCHECK 2 vector to construct psiCHECK2-mTOR 3'-UTR. The empty vector psiCHECK2 was used as a control. When AGS cells were transfected with miRNA-199a-3p mimic and psiCHECK2-mTOR 3'-UTR, luciferase expression was significantly reduced to $50 \%$ of that in cells transfected with the miRNA-199a-3p mimic negative control and psiCHECK2-mTOR 3'-UTR or the miRNA-199a-3p mimic and psiCHECK2 control vector, indicating that the observed elevated expression of miRNA-199a-3p is correlated with a decrease in the luciferase expression level, likely owing to the destabilization of luciferase mRNA resulting from the binding of miRNA199a-3p to the mTOR 3'-UTR (Figure 4). Luciferase activity was, however, highest in cells transfected with the miRNA-199a-3p inhibitor and psiCHECK2-mTOR 3'-UTR. In addition, there was no significant difference in luciferase activity when cells were transfected with the psiCHECK2 control plasmid.

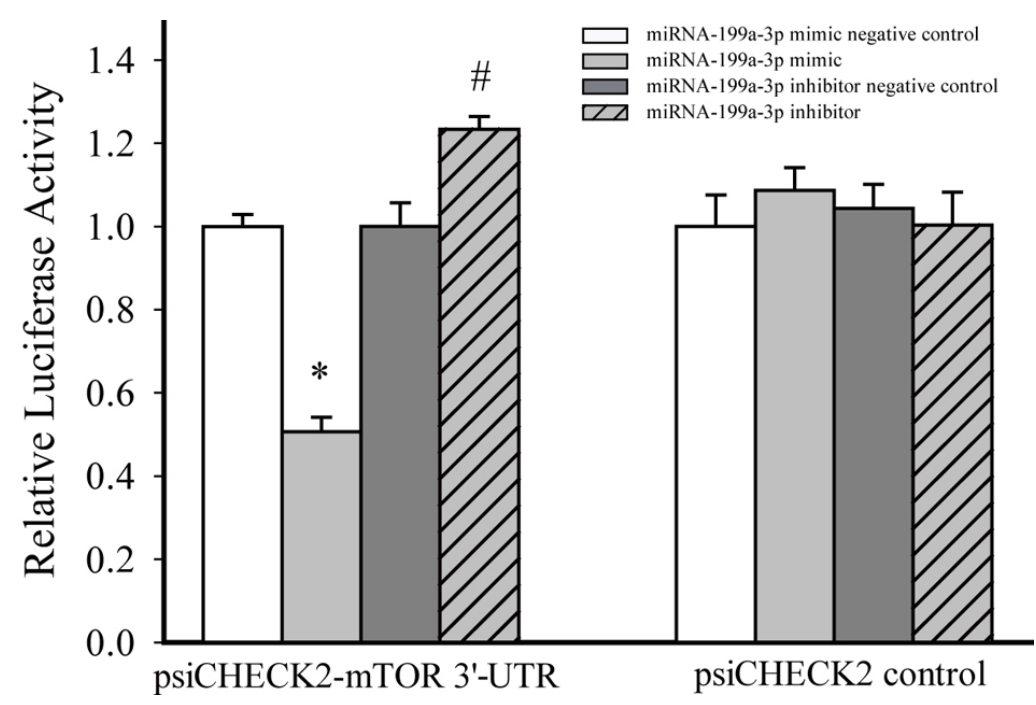

Figure 4. miRNA-199a-3p negatively regulated mTOR by binding to the mTOR 3'-UTR. mTOR 3'-UTR luciferase reporter assay showed that the luciferase activity was significantly reduced in cells co-transfected with miRNA199a-3p mimic and enhanced in cells co-transfected with miRNA-199a-3p inhibitor. Data are reported as means \pm SD. $* \mathrm{P}<0.001$, compared with cells co-transfected with the miRNA-199a-3p mimic negative control. ${ }^{*} \mathrm{P}<0.05$, compared with cells co-transfected with the miRNA-199a-3p inhibitor negative control.

\section{DISCUSSION}

The abnormal expression of various miRNAs has been reported for several leukemias and solid cancers (Calin et al., 2005; Iorio et al., 2005; Volinia et al., 2006). Alteration in the expression levels of miRNAs is highly associated with the progression and prognosis of human malignant diseases (Calin et al., 2005; Schetter et al., 2008). With respect to the func- 
tional mechanism, accumulating evidence indicates that miRNAs behave as tumor oncogenes or suppressors and, thus, play essential roles in human cancer (Cho, 2007).

The expression of miRNA-199a-3p has been previously reported to be decreased in several human malignancies including bladder, breast, liver, and osteosarcoma (Iorio et al., 2005; Jiang et al., 2008; Ichimi et al., 2009; Duan et al., 2011). Here, we showed that its expression was also significantly reduced in human gastric cancer tissues and cells. We also revealed that it can inhibit the proliferation of human gastric cancer cells.

Identification of cancer-specific miRNAs and their targets is critical for understanding their role in tumorigenesis (Iorio et al., 2005). To clarify the mechanism by which miRNA199a-3p inhibited the cell growth of AGS cells, we choose to investigate several potential target genes. Among them, $m T O R$ has been previously reported to be a key promoter of cell growth and a target of miRNA-199a-3p (Fornari et al., 2010). Akt and bcl-2, which are involved in the PI3K signaling pathway, were also studied. Our findings revealed that $m T O R$ is a functional target of miRNA-199a-3p in AGS cells. Over-expression of the miRNA-199a-3p mimic reduced the expression of $m T O R$, while specific inhibition of miRNA-199a-3p increased the expression of $m T O R$. Furthermore, the expression of a luciferase reporter containing $m T O R$ 3'-UTR was attenuated when miRNA-199a-3p was over-expressed. These data, therefore, suggest that the growth-inhibitory effect of miRNA-199a-3p on gastric cancer cells is mediated by the suppression of $m T O R$ expression. Our results also imply that the interaction between miR-199a-3p and mTOR may play a role in the pathogenesis of gastric cancer.

\section{CONCLUSION}

Our results suggest that miRNA-199a-3p is associated with human gastric cancer through its ability to decrease the proliferation of cancer cells by targeting the mTOR signaling pathway. Although the mechanisms behind this targeted inhibition need to be further explored, our results suggest that the described interaction between miRNA-199a-3p and $m T O R$ may have an essential role in gastric cancer development and progression. Taken together, we propose that miRNA-199a-3p is a candidate tumor suppressor miRNA in gastric cancer and, therefore, may be a potential therapeutic target for the treatment of human gastric cancer.

\section{REFERENCES}

Ambros V (2004). The functions of animal microRNAs. Nature 431: 350-355.

Bartel DP (2004). MicroRNAs: genomics, biogenesis, mechanism, and function. Cell 116: 281-297.

Bartel DP (2009). MicroRNAs: target recognition and regulatory functions. Cell 136: 215-233.

Bentwich I, Avniel A, Karov Y, Aharonov R, et al. (2005). Identification of hundreds of conserved and nonconserved human microRNAs. Nat. Genet. 37: 766-770.

Brennecke J, Hipfner DR, Stark A, Russell RB, et al. (2003). bantam encodes a developmentally regulated microRNA that controls cell proliferation and regulates the proapoptotic gene hid in Drosophila. Cell 113: 25-36.

Calin GA, Ferracin M, Cimmino A, Di Leva G, et al. (2005). A MicroRNA signature associated with prognosis and progression in chronic lymphocytic leukemia. N. Engl. J. Med. 353: 1793-1801.

Cho WC (2007). OncomiRs: the discovery and progress of microRNAs in cancers. Mol. Cancer 6: 60.

Duan Z, Choy E, Harmon D, Liu X, et al. (2011). MicroRNA-199a-3p is downregulated in human osteosarcoma and regulates cell proliferation and migration. Mol. Cancer Ther. 10: 1337-1345.

Dyrskjøt L, Ostenfeld MS, Bramsen JB, Silahtaroglu AN, et al. (2009). Genomic profiling of microRNAs in bladder cancer: miR-129 is associated with poor outcome and promotes cell death in vitro. Cancer Res. 69: 4851-4860.

Engels BM and Hutvagner G (2006). Principles and effects of microRNA-mediated post-transcriptional gene regulation. 
Oncogene 25: 6163-6169.

Fornari F, Milazzo M, Chieco P, Negrini M, et al. (2010). MiR-199a-3p regulates mTOR and c-Met to influence the doxorubicin sensitivity of human hepatocarcinoma cells. Cancer Res. 70: 5184-5193.

Gandellini P, Folini M, Longoni N, Pennati M, et al. (2009). miR-205 Exerts tumor-suppressive functions in human prostate through down-regulation of protein kinase Cepsilon. Cancer Res. 69: 2287-2295.

Garzon R, Calin GA and Croce CM (2009). MicroRNAs in Cancer. Annu. Rev. Med. 60: 167-179.

Hatfield S and Ruohola-Baker H (2008). microRNA and stem cell function. Cell Tissue Res. 331: 57-66.

Hohenberger P and Gretschel S (2003). Gastric cancer. Lancet 362: 305-315.

Ichimi T, Enokida H, Okuno Y, Kunimoto R, et al. (2009). Identification of novel microRNA targets based on microRNA signatures in bladder cancer. Int. J. Cancer 125: 345-352.

Iorio MV, Ferracin M, Liu CG, Veronese A, et al. (2005). MicroRNA gene expression deregulation in human breast cancer. Cancer Res. 65: 7065-7070.

Jiang J, Gusev Y, Aderca I, Mettler TA, et al. (2008). Association of MicroRNA expression in hepatocellular carcinomas with hepatitis infection, cirrhosis, and patient survival. Clin. Cancer Res. 14: 419-427.

Kent OA and Mendell JT (2006). A small piece in the cancer puzzle: microRNAs as tumor suppressors and oncogenes. Oncogene 25: 6188-6196.

Lee JW, Choi CH, Choi JJ, Park YA, et al. (2008). Altered MicroRNA expression in cervical carcinomas. Clin. Cancer Res. 14: 2535-2542.

Lewis BP, Burge CB and Bartel DP (2005). Conserved seed pairing, often flanked by adenosines, indicates that thousands of human genes are microRNA targets. Cell 120: 15-20.

Lim LP, Lau NC, Garrett-Engele P, Grimson A, et al. (2005). Microarray analysis shows that some microRNAs downregulate large numbers of target mRNAs. Nature 433: 769-773.

Martin RC, Jaques DP, Brennan MF and Karpeh M (2002). Extended local resection for advanced gastric cancer: increased survival versus increased morbidity. Ann. Surg. 236: 159-165.

O’Donnell KA, Wentzel EA, Zeller KI, Dang CV, et al. (2005). c-Myc-regulated microRNAs modulate E2F1 expression. Nature 435: 839-843.

Schetter AJ, Leung SY, Sohn JJ, Zanetti KA, et al. (2008). MicroRNA expression profiles associated with prognosis and therapeutic outcome in colon adenocarcinoma. JAMA 299: 425-436.

Su H, Yang JR, Xu T, Huang J, et al. (2009). MicroRNA-101, down-regulated in hepatocellular carcinoma, promotes apoptosis and suppresses tumorigenicity. Cancer Res. 69: 1135-1142.

Takagi T, Iio A, Nakagawa Y, Naoe T, et al. (2009). Decreased expression of microRNA-143 and -145 in human gastric cancers. Oncology 77: 12-21.

Tsujiura M, Ichikawa D, Komatsu S, Shiozaki A, et al. (2010). Circulating microRNAs in plasma of patients with gastric cancers. Br. J. Cancer 102: 1174-1179.

Tsukamoto Y, Nakada C, Noguchi T, Tanigawa M, et al. (2010). MicroRNA-375 is downregulated in gastric carcinomas and regulates cell survival by targeting PDK1 and 14-3-3zeta. Cancer Res. 70: 2339-2349.

Ueda T, Volinia S, Okumura H, Shimizu M, et al. (2010). Relation between microRNA expression and progression and prognosis of gastric cancer: a microRNA expression analysis. Lancet Oncol. 11: 136-146.

Volinia S, Calin GA, Liu CG, Ambs S, et al. (2006). A microRNA expression signature of human solid tumors defines cancer gene targets. Proc. Natl. Acad. Sci. U. S. A. 103: 2257-2261.

Wu Q, Jin H, Yang Z, Luo G, et al. (2010). MiR-150 promotes gastric cancer proliferation by negatively regulating the pro-apoptotic gene EGR2. Biochem. Biophys. Res. Commun. 392: 340-345.

Yanaihara N, Caplen N, Bowman E, Seike M, et al. (2006). Unique microRNA molecular profiles in lung cancer diagnosis and prognosis. Cancer Cell 9: 189-198.

Zamore PD and Haley B (2005). Ribo-gnome: the big world of small RNAs. Science 309: 1519-1524. 